\author{
M. Ledl · J. Hohenecker • C. Francesconi - I. Roots • \\ M. F. Bauer - M. Roden
}

\title{
Acute myopathy in a type 2 diabetic patient on combination therapy with metformin, fenofibrate and rosiglitazone
}

Received: 22 March 2005 / Accepted: 7 June 2005 / Published online: 17 August 2005

(C) Springer-Verlag 2005

\begin{abstract}
Aims/hypothesis: This report describes the case of a 75-year-old male type 2 diabetic Caucasian who was admitted to the clinical ward because of acute pain and cramps in both calf muscles. Materials and methods: Neuromuscular function was assessed by electromyography and electroneurography of the right leg. An open biopsy was taken from the left vastus lateralis muscle for histological and histochemical analyses. Southern blotting was performed to detect defects in mitochondrial DNA and tRNA. Cytochrome P450 (CYP-P450) polymorphisms were analysed in blood cells. Results: Fifteen weeks before admission, the patient's lipid-lowering medication was switched from simvastatin to fenofibrate because of predominant hypertriglyceridaemia; this did not affect creatine kinase levels. Three weeks before admission, rosiglitazone was added to his existing metformin therapy because of worsening metabolic control. Upon admission, serum enzymes indicating myopathy were elevated (creatine kinase 6897 $\mathrm{U} / 1$, myoglobin $902 \mathrm{ng} / \mathrm{ml}$ ) and kidney function was impaired (creatinine $0.116 \mathrm{mmol} / \mathrm{l}$, blood urea nitrogen $2.3 \mathrm{mmol} / \mathrm{l})$. Electrophysiology revealed myopathy and sensory polyneuropathy. Histology showed multiple damage of the myofibrillar architecture. There was no evi-
\end{abstract}

\footnotetext{
M. Ledl $\cdot$ J. Hohenecker $\cdot$ M. Roden $(\bowtie)$

First Medical Department, Hanusch Hospital,

Heinrich Collin Strasse 30,

A-1140 Vienna, Austria

e-mail: michael.roden@wgkk.sozvers.at

Tel.: +43-1910-2185011

Fax: +43-1910-2185019

C. Francesconi

Department of Public Health,

Vienna, Austria

I. Roots

Institute of Clinical Pharmacology,

University Hospital Charité,

Berlin, Germany

M. F. Bauer

Institute of Clinical Chemistry,

Munich-Schwabing Hospital,

Munich, Germany
}

dence of defects in mitochondrial DNA or tRNA. Furthermore, no functional limitations in CYP2C9, CYP2C19 and CYP2D6 were detected. Following withdrawal of the oral medication and intravenous hydration, clinical symptoms and laboratory parameters gradually decreased. Conclusions/ interpretation: Until more data from controlled trials are available, we recommend that combination therapy with fibrates and thiazolidinediones should be monitored frequently by measurements of serum creatine kinase and creatinine, specifically in patients with pre-existing nephropathy and polyneuropathy.

Keywords Glitazones - HMG CoA reductase inhibitors · Hydroxymethylglutaryl coenzyme A reductase inhibitors . Mitochondrial myopathy - Oral hypoglycaemic drugs ·

Renal failure $\cdot$ Rhabdomyolysis · Statin · Thiazolidinedione $\cdot$ Type 2 diabetes mellitus

Abbreviations CK: creatine kinase $\cdot$ CYP: cytochrome P

\section{Introduction}

Since multifactorial intervention has proven to reduce morbidity and mortality in controlled prospective studies, such as the UK Prospective Diabetes Study (UKPDS) and the Steno-2 study, polypragmatic pharmacotherapy has been recommended and increasingly used in type 2 diabetic patients. In particular, oral hypoglycaemic and lipid-lowering drugs are frequently prescribed in combination therapy. However, this could also increase the risk and frequency of adverse events.

Lipid-lowering medication in monotherapy and, more likely, in combination therapy, e.g. statins (hydroxymethylglutaryl coenzyme A reductase inhibitors) plus fibrates, can cause different degrees of myopathy, ultimately leading to rhabdomyolysis and acute renal failure [1]. Newer oral hypoglycaemic drugs, such as thiazolidinediones (glitazones), are now used to treat hyperglycaemia in type 2 diabetic patients and cause side effects that include body weight gain, mostly due to fluid retention [2]. Elevation of liver transaminases might occur, but severe hepatotoxicity is currently proven 
only for troglitazone, which was therefore withdrawn from the market some years ago.

\section{Case report}

A 75-year-old male Caucasian was admitted to the ward because of acute onset of pain and cramps in both calf muscles, particularly during body movements. He neither had a recent history of muscular injuries, viral infections or heavy physical exercise nor a family history of hereditary neuromuscular diseases. Clinical and neurological examination did not reveal gross abnormalities. Serum enzymes indicating myopathy were markedly elevated (creatine kinase $[\mathrm{CK}]$ 6,897 U/l, normal range 0-171 U/l; myoglobin $902 \mathrm{ng} / \mathrm{ml}$, normal range $0-110 \mathrm{ng} / \mathrm{ml}$ ) (Fig. 1), the CK isoenzyme pattern being consistent with skeletal muscle origin (CK-MB 1\%, normal range 0-6\%). Lactate dehydrogenase was also elevated $(285 \mathrm{U} / \mathrm{l}$; normal range 50-248 U/1). Parameters of kidney (serum creatinine $0.116 \mathrm{mmol} / \mathrm{l}$, normal range $0.03-0.07 \mathrm{mmol} / 1$; blood
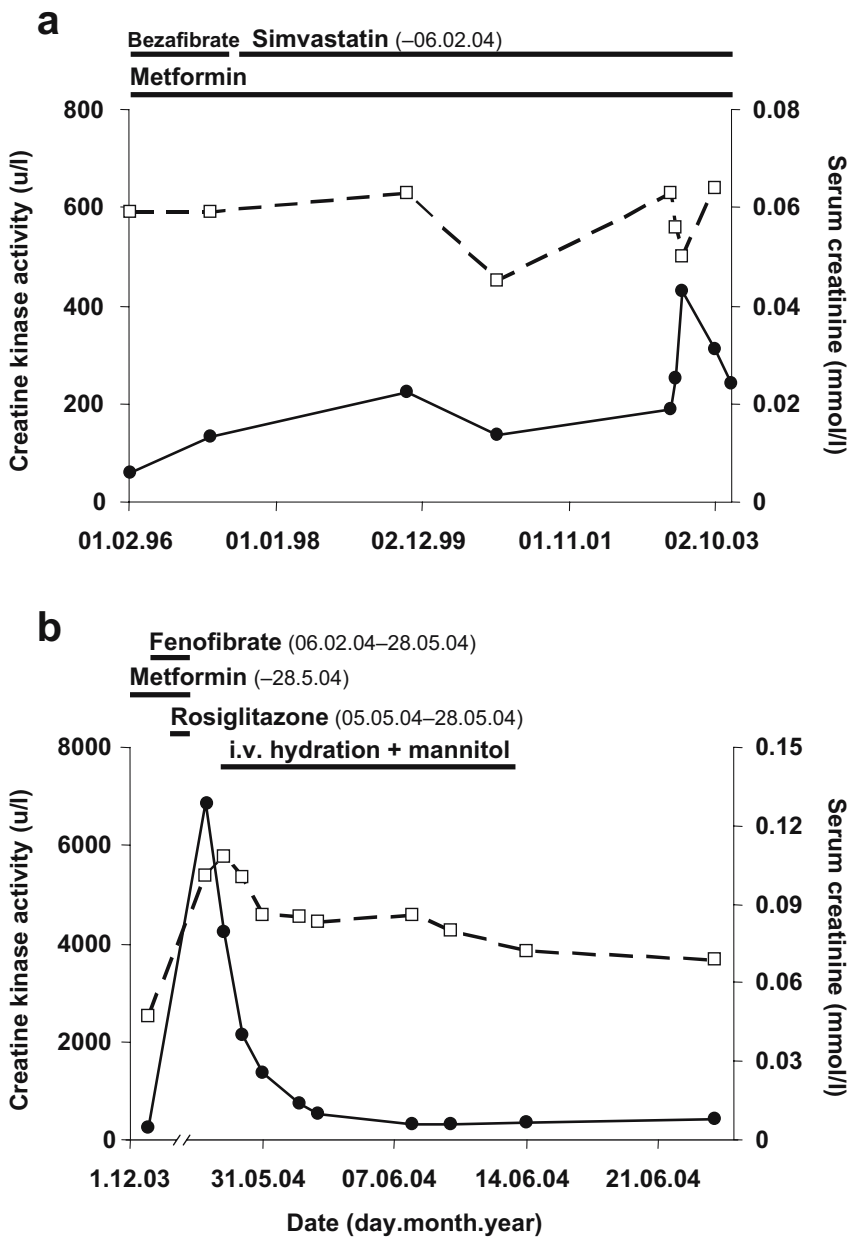

Fig. 1 Time course of serum creatine kinase activity (solid circles) and creatinine (open squares) and the use of oral hypoglycaemic and lipid-lowering medications (as indicated above each graph) from 1 February 1996 to 15 December 2003 (a) and from 1 December 2003 to 24 June 2004 (b) in a 75-year-old male patient with type 2 diabetes, who developed acute myopathy in May 2004 urea nitrogen $2.3 \mathrm{mmol} / \mathrm{l}$, normal range $0.3-1.3 \mathrm{mmol} / \mathrm{l}$ ) and liver function (glutamate oxaloacetate transaminase $68 \mathrm{U} / 1$, normal range 2-25 U/1; glutamate pyruvate transaminase $49 \mathrm{U} / 1$, normal range 2-45 U/l) were increased (Fig. 1). Hypothyroidism as a potential risk factor for myopathy was excluded by the patient's normal thyroidstimulating hormone concentration.

The patient had an 11-year history of type 2 diabetes mellitus, together with hypertension, hyperlipidaemia and hepatic steatosis. He had had diabetic neuropathy with mild asymptomatic CK elevation, ranging from 250 to $350 \mathrm{U} / 1$, for 4 years, documented by electroneurography 4 months before admission (Fig. 1a). Nine years ago he had received cardiac bypass surgery and a mechanical aortic valve.

His drug treatment consisted of phenprocoumon, valsar$\tan$ (an angiotensin receptor blocker) and inhaled sympathomimetics because of obstructive airway disease. Until a few months before admission, the patient had been in good metabolic control with metformin (1,000 mg twice daily) monotherapy (Fig. 1a). Fifteen weeks before admission, lipid-lowering medication with simvastatin (20 mg daily) was switched to fenofibrate ( $200 \mathrm{mg} /$ day) because of predominant hypertriglyceridaemia, which probably resulted from increased insulin resistance (Fig. 1b). Twelve weeks after the first dose of fenofibrate, rosiglitazone $(2 \mathrm{mg}$ twice daily) was added to the ongoing therapy by an out-patient department because of worsening of glycaemic control $\left(\mathrm{HbA}_{1} \mathrm{c}>7.0 \%\right)$.

Twenty-three days later the patient was referred to our hospital. Electromyography of the right rectus femoris muscle showed no spontaneous muscular activity and the interference pattern indicated myopathy. Electroneurography recorded no sensory potentials from the peroneal nerves, which was consistent with polyneuropathy. Peripheral vascular disease and cardiomyopathy were excluded by duplex sonography and transthoracic echocardiography. After discontinuation of oral anticoagulation, open biopsy of the left vastus lateralis muscle revealed multiple damage of the myofibrillar architecture and scattered defects in oxidative enzymes activities. While inflammatory cells and ragged red fibres were absent, a single cyclooxygenase-negative muscle fibre was detected, suggesting a metabolic disorder without proof of toxic myopathy.

As thiazolidinediones, such as rosiglitazone, are metabolized by cytochrome $\mathrm{P} 450$ and can cause mitochondrial dysfunction [3, 4], further analyses were performed. Southern blotting revealed neither a deletion/insertion in the mitochondrial DNA nor common tRNA mutations $(3243,3250,3271,8344)$, or 7472 Cins, 7497 and 7512 in tRNA Ser(UCN), all of which are associated with mitochondrial myopathies. No functional limitations associated with frequent polymorphisms in CYP-P4502C9, CYP2C19 and CYP2D6 were detected.

Upon the patient's admission to hospital, metformin was withdrawn because of impaired renal function. Fenofibrate and rosiglitazone were discontinued because of suspected drug interaction. During 13 days of intravenous treatment with hydration and mannitol, muscle enzymes and creatinine decreased continuously to values observed before 
rosiglitazone treatment (Fig. 1b) and muscular symptoms vanished in parallel. A fat-reduced diet plus low-dose gliclazide resulted in near-normoglycaemia.

\section{Discussion}

The present case indicates that simultaneous treatment with fenofibrate and rosiglitazone might lead to severe myopathy in diabetic patients who are already at increased risk because of pre-existing polyneuropathy and impaired renal function. Severe myopathy and rhabdomyolysis are potentially life-threatening and require hospitalisation and intensive treatment.

Such side effects are well known for combination therapy with statin plus fibrate and have been attributed to a pharmacokinetic interaction with CYP-P450 [1, 4-6]. Thiazolidinediones (troglitazone, rosiglitazone, pioglitazone) effectively reduce hyperglycaemia but may cause side effects [2]. Although troglitazone was withdrawn from the market because of hepatotoxicity, there was also a report of myopathy [7]. Levels of CK were reported to have increased to $10,570 \mathrm{U} / 1$ in a 59-year-old type 2 diabetic man with diabetic retinopathy and neuropathy, 6 months after the addition of troglitazone $(400 \mathrm{mg} /$ day $)$ to his existing therapy with glibenclamide. As mild alcoholic liver injury was also diagnosed, the authors suggested that ethanol, a potent inducer of CYP-P450, could have amplified the muscle toxicity of troglitazone. Of note, our patient denied daily consumption of alcohol but had elevated liver transaminases, probably as a result of non-alcoholic fatty liver disease.

Although this adverse effect is not widely known for newer thiazolidinediones [8], one case of asymptomatic transient CK elevation was identified in a multicentre trial after simvastatin had been added to thiazolidinedione therapy [9]. Our patient had also been on statin therapy for years, without clinical signs of myopathy. After replacement of statin with fenofibrate, his drug records were repeatedly checked and confirmed that he was not taking any statin for almost 4 months before he developed myopathy. Thus, and because rhabdomyolysis due to lipid-lowering drugs mostly occurs with cerivastatin alone or in combination with gemfibrozil [1], statins can be largely excluded as a cause of myopathy in our patient.

Another case of acute rhabdomyolysis occurred in a 56-year-old female type 2 diabetic patient when fenofibrate (200 mg daily) was added to her glucose-lowering therapy with pioglitazone, metformin and NPH insulin [6]. Like our patient, this woman also featured hyperlipidaemia and polyneuropathy. Symptoms, CK elevation exceeding 23,000 U/1 and impairment of kidney function, started 10 days after initiation of fenofibrate and returned to baseline within weeks after discontinuation of fenofibrate and metformin. Of note, our patient had been on fibrate therapy for years without clinical myopathy, and then received the identical dose of fenofibrate for months before admission. Because of the short interval between the introduction of rosiglitazone and the onset of clinical symptoms, and the simultaneous development of liver and kidney dysfunction, we decided to discontinue not only fenofibrate and metformin but also rosiglitazone treatment. Thus, we cannot rule out the possibility that either fenofibrate or rosiglitazone was exclusively responsible for the myopathy of our patient and that withdrawal of fenofibrate - along with metformin because of impaired renal function - could have been sufficient to cure our patient.

It is of note that all patients who developed myopathy or rhabdomyolysis during thiazolidinedione treatment $[6,7$, this report] suffered from diabetic neuropathy. Our patient featured an asymptomatic mild CK elevation for some years, indicating pre-existing myopathy as a consequence of long-term diabetic neuropathy. This and other conditions, such as non-alcoholic fatty liver, could have made the patient specifically sensitive to the observed adverse effect. Despite the absence of known genetic risk factors, the combination of glucose-lowering drugs we have described could accelerate and worsen an existing myopathy under such conditions.

We would recommend that, until data from further studies are available, combination therapy using fibrates with thiazolidinediones should be monitored by frequent measurement of CK and creatinine specifically in patients with nephropathy and polyneuropathy, who are at increased risk of developing further muscular alterations.

Acknowledgements We would like to thank Professor Dr Herbert Budka and the staff of the Clinical Institute for Neurology, Medical University of Vienna, for the preparation and histological examination of the muscle biopsy.

\section{References}

1. Alawi AA, Kuvin JT, Karas RH (2004) Risk of adverse events with fibrates. Am J Cardiol 94:935-958

2. Yki-Jarvinen H (2004) Thiazolidinediones. N Engl J Med 351:1106-1118

3. Niemi M, Backman JT (2004) Effects of trimethoprim and rifampicin on the pharmacokinetics of the cytochrome P450 2C8 substrate rosiglitazone. Clin Pharmacol Ther 76:239-249

4. Scatena R, Martorana G (2004) Mitochondrial dysfunction by synthetic ligands of peroxisome proliferator activated receptors (PPARs). IUBMB Life 56:477-482

5. Bellosta S, Paoletti R, Corsini A (2004) Safety of statins: focus on clinical pharmacokinetics and drug interactions. Circulation 109 (Suppl 1):11150-11157

6. Barker BJ, Goodenough RR, Falko JM (2003) Fenofibrate monotherapy induced rhabdomyolysis. Diabetes Care 26:24822483

7. Yokoyama M, Izumiya Y (2000) Acute rhabdomyolysis associated with troglitazone. Diabetes Care 23:421-422

8. Harmel AL, Kendall DM, Buse JB, Boyle PJ, Marchetti A, Lau H (2004) Impact of adjunctive thiazolidinedione therapy on blood lipid levels and glycemic control in patients with type 2 diabetes. Curr Med Res Opin 20:215-223

9. Lewin AJ, Kipnes MS, Meneghini LF et al (2004) Effects of simvastatin on the lipid profile and attainment of low-density lipoprotein cholesterol goals when added to thiazolidendione therapy in patients with type 2 diabetes mellitus: a multicenter, randomized, double-blind, placebo-controlled trial. Clin Ther 26:279-289 